\section{LAS TEMÁTICAS Y PREOCUPACIONES DE LAS INVESTIGADORAS ÉLITE EN CIENCIAS SOCIALES DE LAS UNIVERSIDADES CATALANAS}

\author{
Marina Tomàs Folch \\ Universidad Autónoma de Barcelona \\ Marina.Tomas@uab.cat \\ Trinidad Mentado Labao \\ Universidad de Barcelona \\ trini.mentado@ub.edu
}

\section{TOPICS AND CONCERNS OF THE ELITE AMONG SOCIAL SCIENCES RESEARCHERS OF THE CATALAN UNIVERSITIES}

\begin{abstract}
Cómo citar este artículo/ Citation: Tomàs Folch, M. y Mentado Labao, T. (2013). Las temáticas y preocupaciones de las investigadoras élite en Ciencias Sociales de las universidades catalanas. Arbor, 189(760):a019. doi: http://dx.doi.org/10.3989/ arbor.2013.760n2005
\end{abstract}

Recibido: 25 abril 2012; Aceptado: 22 noviembre 2012.

RESUMEN: Las Ciencias Sociales son áreas del conocimiento en las cuales inciden de forma muy importante las circunstancias que rodean al personal investigador. Los enfoques que se adoptan ante el tópico a investigar, las metodologías o maneras de estudiarlo, son especialmente claves en estas áreas. Entre estas circunstancias se encuentra el género de la investigadora principal.

La metodología elegida para abordar este estudio ha sido cualitativa. En concreto, las entrevistas focalizadas a nivel individual y el análisis del discurso en la producción científica son las técnicas utilizadas.

De los resultados obtenidos podemos anunciar tendencias más que conclusiones. Sólo una cuarta parte de los grupos son liderados por mujeres y éstas parece que tienen unas condiciones especiales y que están ahí porque siguen las pautas investigadoras al uso. Parece observarse como rasgo diferenciador del género femenino una mayor preocupación por la formación del equipo de investigadores y por el clima del grupo.

PALABRAS CLAVE: Mujeres investigadoras; Ciencias Sociales y Universidad.
Copyright: (c) 2013 CSIC. Este es un artículo de acceso abierto distribuido bajo los términos de la licencia Creative Commons Attribution-Non Commercial (by-nc) Spain 3.0.

ABSTRACT: The Social Sciences are areas of knowledge in which the researcher's context can have a major significance. The approaches taken to the research topic and the methodologies of study are the key elements in these areas. The gender of the principal investigator is one of the significant factors.

A qualitative methodology has been chosen for this study. Specifically, the techniques that used are individual interviews and discourse analysis in scientific production.

Our results reveal a number of trends rather than firm conclusions. Only a quarter of the groups are led by women, whose circumstances seem to be somewhat special and whose position is due to the use of average research models. A greater concern for the training of the research team and for the mood within the group seems to be a differential feature of the female gender.

KEYWORDS: Women researchers; Social Sciences and University. 


\section{INTRODUCCIÓN}

Presentamos en este artículo parte de un trabajo más amplio co-subvencionado por el Institut de les Dones de la Generalitat de Catalunya (Ref. U-45/1) finalizado en el año 2011 con el título de "La investigación académica en Ciencias Sociales en las universidades catalanas desde una perspectiva de género. Estudio de caso de la Universidad Autónoma de Barcelona (UAB) y de la Universidad de Barcelona (UB)", donde destacamos algunos de los resultados obtenidos relacionados con las temáticas y preocupaciones de las élites académicas en la investigación de Ciencias Sociales (CCSS).

Comenzamos con una breve contextualización sobre la situación en la que se encuentran las universidades españolas y cómo éstas asumen el compromiso de velar por la igualdad entre mujeres y hombres. Seguimos con un análisis de la situación de la investigación en el ámbito de las CCSS y sus principales preocupaciones en relación a la producción científica, la evaluación y la financiación. Posteriormente, la presencia de la mujer en la universidad nos pone en alerta sobre la existencia del llamado "techo de cristal" que como espacio superior invisible puede suponer un obstáculo a la mujer investigadora. Para finalizar, damos cuenta de la metodología llevada a cabo, así como de los resultados y de discusión con la información obtenida y las conclusiones.

\section{LA UNIVERSIDAD ANTE LA IGUALDAD ENTRE MUJE- RES Y HOMBRES}

Actualmente, las universidades españolas pretenden colocarse en una mejor posición para la cooperación interna y la competencia internacional, creando, transmitiendo, desarrollando y produciendo conocimiento científico y tecnológico y de transferencia a la sociedad, todo ello con el fin de ser más atractivas en la sociedad actual y en el mundo globalizado. Una de las medidas adoptadas, además de los cambios funcionales y estructurales, es lograr una sociedad más tolerante e igualitaria, en la que se respete la igualdad entre hombres y mujeres. De esta manera, la (LOMLOU, 2007) hace alusión directa a la "Paridad en la representación de los órganos de gobierno para garantizar la igualdad de oportunidades y no discriminación por razones de sexo, tanto para docentes como estudiantes y personal de administración y servicio". En la misma ley se fomenta la igualdad en la investigación, así como la creación de estructuras específicas que en el seno de las universidades garanticen el desarrollo de funciones destinadas al principio de igualdad entre mujeres y hombres.

En relación a la política nacional de igualdad en España (Ley Orgánica 3/2007, de 22 de marzo, para la Igualdad Efectiva de Mujeres y Hombres), el Ministe- rio de Educación desempeña un papel fundamental en el desarrollo de políticas en pos de esa igualdad. Así se diseña un conjunto de acciones a desarrollar en los próximos años, a la vez que se perfilan algunas normas legales como la Ley de la Ciencia, la Tecnología y la Innovación. Un ejemplo de ello es la creación de la Unidad de Mujeres y Ciencia (UMYC) creada desde el Ministerio de Educación (MEC) y adscrita al Ministerio de Ciencia e Innovación como el órgano encargado de poner en práctica el principio de transversalidad de género, o mainstreaming, en los ámbitos científico, tecnológico y de innovación. Con ello, pretende promover una presencia de las mujeres en todos los ámbitos del sistema de ciencia, tecnología e innovación; promover la inclusión del género como categoría transversal en la investigación científica y en los desarrollos tecnológicos y la innovación.

Entre las notas biográficas del Consejo Superior de Investigaciones Científicas (CSIC) es posible conocer mujeres ilustres que han pasado a la historia de la academia por sus legados pero todas ellas corresponden al ámbito de conocimiento de las ciencias llamadas duras -desde la química a la medicina-. El estudio de la élite académica es importante, no sólo porque forma parte de la sociología del poder, como apunta García de León, 2001, sino porque además el estudio de la élite académica puede relacionarse directamente con lo que llamamos "el cambio social". Las élites son la "punta del iceberg" (García de León, 2001) de los procesos de cambio. Los estudios sobre la producción de conocimiento permiten ver las cualidades de los diferentes productos, los orígenes ideológicos de los discursos subyacentes y los procesos sociales que participan en su configuración.

En los últimos años algunas aproximaciones a la investigación desde la perspectiva de género han ayudado a esclarecer como mínimo la distribución por sexos del personal investigador (Pérez Sedeño 2001, 2004, 2005; Arbor 2002, Miqueo et al., 2008), así como las categorías profesionales que ocupan globalmente las mujeres y hombres aunque hasta el momento carecemos de datos mínimamente fiables sobre la productividad e impacto de su trabajo. En el caso de la universidad actual catalana, podemos afirmar que ha vivido en los últimos años cambios importantes pero que este proceso no ha ido acompañado por un cambio en la distribución igualitaria entre hombres y mujeres en el ámbito de la investigación en general y en CCSS en particular.

\section{LA INVESTIGACIÓN EN LAS CIENCIAS SOCIALES}

La actividad investigadora es una de las principales tareas que tiene el personal académico en la Universidad. Ésta requiere de unas competencias, por las cuáles se forma especialmente al profesorado universita- 
rio y que finaliza con el título de Doctor. Ahora bien, ¿es lo mismo investigar en Ciencias Experimentales que en Ciencias Tecnológicas que en Ciencias Humanas o Sociales? Del Pozo (2010) sostiene que no tiene sentido desde un punto de vista epistemológico hacer una distinción antagónica entre las culturas científicotecnológicas y las social-humanísticas. Los elementos de racionalidad, strictu senso, que se usan en Ciencias Naturales no son suficientes para investigar en CCSS. En opinión de Subirats (2010) las CCSS se interesan por lo particular y no sólo por lo general y se busca entender las formas en que funciona el mundo real, muchas veces impredecible. Una buena investigación en CCSS debe ser capaz de explicar no sólo lo que pasa sino las implicaciones sociales y políticas que supone y el grado de consenso o conflicto social que genera. La teoría que se deriva de este conocimiento difícilmente podrá ser situada y valorada al mismo nivel que la que se produce en el ámbito de las Ciencias Naturales.

Botella (2010) advierte que junto al reconocimiento de las singularidades de la investigación en CCSS también en esta área el investigador/a debe perseguir un ideal universalista, debe hacer llegar sus resultados a toda la comunidad científica sea esta de la misma disciplina o de otra. Las CCSS no son disciplinas donde la investigación que se desarrolla sea totalmente homogénea. Dentro de CCSS hay un gran número de disciplinas muy diferenciadas y con particularidades muy notables y dimensiones variables respecto a la actividad investigadora. Entendemos que se pueden considerar como disciplinas de CCSS las siguientes: Derecho, Economía, Geografía, Historia, PedagogíaEducación, Psicología, Sociología. Ahora bien desde una perspectiva organizativa de la investigación sí que existen ciertas prácticas que merecen ser estudiadas o tenidas en cuenta. ¿Existe mayor tendencia a investigar individualmente o en grupos pequeños en CCSS? ¿Dónde se investiga? ¿Cuál es el laboratorio de CCSS? ¿Qué presupuestos se manejan? Estas circunstancias de las CCSS fueron tratadas por Panchón (2010) en el taller sobre la evaluación de la investigación en CCSS y Humanidades llevado a cabo por el AQU:

- La ambigüedad de los límites entre CCSS y Humanidades y cuáles son las áreas que pertenecen a una u otra.

- El estilo propio de investigar en CCSS debido a la complejidad de los fenómenos sociales y educativos.

- El debate ideológico que acompaña a dicha investigación por la importancia que tiene el acercamiento desde una u otra perspectiva al estudiar un fenómeno social.

- La necesidad de revisar los criterios de valoración existentes sobre evaluación de la actividad investigadora y el impacto de ésta.
Los grupos de investigación denominados SGR (Grupos de Investigación Consolidados) son grupos reconocidos por la Agencia de Gestión de Ayudas Universitarias y de Investigación (AGAUR). Esta estructura es singular en el sistema universitario catalán porque no existe en otras del estado español. Estos grupos facilitan el trabajo colectivo e interdisciplinar, y también la inserción de investigadores noveles en el sistema de investigación, la captación de recursos y su optimización. Pertenecer a un grupo con mucha productividad, dónde a menudo el número de autores de los trabajos es elevado, incrementa la posibilidad de recibir citaciones posteriores. Aún así, cuando el sistema actual se vertebra por la vía de los grupos de investigación, existen investigadores individuales, que todavía trabajan más o menos en solitario por las especificidades propias de su área de conocimiento.

Respecto a la producción científica en CCSS hay que señalar que existe una cultura propia caracterizada tradicionalmente por la producción de las monografías, libros y capítulos, uso de lenguas menos universales como el catalán, español, francés o alemán. Ello no ha favorecido la visibilidad y el prestigio de la investigación en CCSS. La investigación en CCSS y su evaluación ha sido objeto de preocupación por parte de la misma comunidad investigadora y las instancias que la administran como las universidades y agencias de calidad desde hace una década como mínimo. En el campo de las Ciencias Experimentales y Tecnológicas ha sido posible y fácil establecer criterios de evaluación y de divulgación de la investigación realizada y la correspondiente producción científica, en CCSS y Humanidades cuesta encontrar puntos de acuerdo y consenso. Así lo demuestran las diversas Jornadas organizadas por la Agencia de Calidad del Sistema Universitario de Cataluña (AQU) en mayo del 2005 y en enero 2010. Una de las conclusiones de estos encuentros ha sido que las políticas de evaluación deberán tener en cuenta el contexto de oportunidad para llevar a cabo la investigación. Las políticas de financiación existentes y la inserción en grupos de investigación potentes son aspectos que pueden condicionar las oportunidades de investigar.

La financiación de la investigación en CCSS pone énfasis en la capacidad de ésta para hacer frente y contribuir a superar problemas sociales presentes y futuros. Pero ¿cómo se financia la investigación en CCSS? Este es un problema mayor si cabe que en otras disciplinas debido al bajo interés que tienen las empresas para financiar dichos proyectos. Esto lleva a plantearse el debate de si hay que financiar la investigación en CCSS con fondos públicos o privados.

\section{LAS MUJERES Y LA INVESTIGACIÓN EN LA UNIVER- SIDAD}

A pesar de que las mujeres españolas obtuvieron el soporte legal necesario para acceder de forma gene- 
ralizada a la universidad en 1910 (Guil, 2004) continúa existiendo serias dificultades para acceder a lugares relevantes y de poder en el mundo académico. Los datos existentes sobre la situación de la mujer en la Ciencia y la Universidad ponen en evidencia las dimensiones de discriminación y desigualdad en el momento de la promoción profesional. La plena incorporación de la mujer a la universidad no ha implicado su promoción en el mundo académico, en concreto en la investigación.

Efectivamente, y así lo expresa el informe "Académicas en cifras 2007" publicado por la Unidad de Mujeres y Ciencia del Ministerio de Educación que pretende servir de instrumento de trabajo para conocer la situación real de las mujeres en el sistema universitario español. Ellas son mayoría entre los titulados en la universidad pública (60\% en el curso 2005-2006), mientras que el número de catedráticas no alcanza el $14 \%$. La presencia de las mujeres en la mayoría de los campos de la vida económica, social y política es cada vez mayor, sin embargo sigue siendo minoritaria en la investigación científica. Aunque la representación femenina en el panorama universitario -sobre todo entre quienes acaban sus estudios universitarios- es cada vez mayor, a medida que va aumentando el escalafón académico el porcentaje se reduce sustancialmente. A nuestro entender son los factores personales y socioculturales los que constituyen el llamado "techo de cristal" académico, que como barrera invisible obstaculiza o ralentiza el acceso a las mujeres a ocupar puestos de élite en las universidades españolas.

De igual manera la mayor presencia de mujeres entre el profesorado universitario ha ido acompañada de una mayor dedicación de éstas a determinadas disciplinas consideradas más "femeninas" (Humanidades y CCSS y Jurídicas). Es también un dato relevante que para aquellas que acceden al escalafón superior en la investigación prefieran profundizar en temas relacionados con la mujer. Efectivamente, entre sus objetivos está la creación de espacios, recursos e iniciativas sobre mujeres o el aumento de la capacidad de integración de éstas.

\section{METODOLOGÍA DE LA INVESTIGACIÓN}

En el estudio más amplio ${ }^{1}$ nos propusimos como objetivo principal de la investigación estudiar al colectivo de investigadoras principales (IP) que dirigen Grupos de Investigación Consolidados (SGR) en CCSS en las universidades catalanas. Más concretamente:

- Conocer los intereses y preocupaciones que sobre la investigación tienen las IP de CCSS.

- Analizar las decisiones metodológicas de los grupos de investigación liderados por mujeres.

- Conocer los elementos que favorecen y los que dificultan la producción científica de las mujeres en la Universidad.

- Conocer el uso del discurso en las producciones científicas de las investigadoras de las universidades estudiadas así como identificar si existen tendencias entre las mujeres profesoras universitarias de Ciencias Sociales que permitan identificar una manera común de expresarse y cuáles son las características principales de su comportamiento discursivo.

En este artículo aportamos los resultados y conclusiones referidos al primero de los objetivos: conocer los intereses y preocupaciones de investigación de las IP de la UAB y UB. Todo ello, con la finalidad de visibilizar la realidad para diagnosticar y comprender la situación de las mujeres investigadoras en CCSS.

El método utilizado en este estudio responde a las premisas del método interpretativo pues se fundamenta en comprender los significados sociales que las personas desarrollan en relación al contexto, los objetos y otras personas. Por tanto, este estudio ha requerido de una metodología cualitativa que de acuerdo con los objetivos planteados, se basó en estrategias etnográficas tales como entrevistas focalizadas y análisis del discurso. Para la realización de las entrevistas tomamos como población dos universidades: Universidad Autónoma de Barcelona y la Universidad de Barcelona. De estas dos instituciones se seleccionaron equipos de investigación dirigidos por profesoras y con el distintivo "Grupo Consolidado de Investigación (SGR)" de la Generalitat de Catalunya de las áreas de Educación, Sociología, Derecho, Económicas y Empresariales (tabla 1).

Tabla 1. Grupos SGR de investigación en Ciencias Sociales

\begin{tabular}{|c|c|c|c|}
\hline & Grupos de Ciencias Sociales & $\begin{array}{c}\text { Grupos liderados por } \\
\text { mujeres }\end{array}$ & Grupos liderados por hombres \\
\hline UAB & 69 & $\mathbf{2 4}$ & 45 \\
\hline UB & 89 & $\mathbf{3 6}$ & 53 \\
\hline
\end{tabular}


El instrumento que nos permite recoger información y datos de manera intensiva y extensiva es la entrevista focalizada. Esta, a diferencia de la entrevista en profundidad, permite un posicionamiento privilegiado al investigador/a por cuanto ya ha podido analizar previamente la situación objeto de la entrevista.

La entrevista se organiza en torno a una serie de cuestiones abiertas de carácter muy general para posteriormente ir centrando el interés en aspectos más específicos. Se diseñan a partir de los tópicos que desde el marco teórico aparecen como principales. Estos serían: los antecedentes y contexto actual de la situación profesional, el rol de la investigación en relación a otras funciones propias de su perfil profesional, los factores de éxito desde la propia percepción, el liderazgo del grupo de investigación, las decisiones metodológicas, los tópicos de investigación en las CCSS y la difusión de resultados de la investigación. Posteriormente, se seleccionan los criterios específicos que suponen ya una primera fase de análisis en la medida que acontecerán codificaciones deductivas. La relación entre tópicos y criterios específicos queda reflejada en la tabla 2.

El análisis de la información procedente de las entrevistas se realizó a través del diseño de un sistema de categorías que combinó las variables específicamente indagadas con otras de carácter emergente. Se utilizó una codificación múltiple: descriptiva e interpretativa y se hizo uso del programa MAXQDA para el procesamiento y análisis del conjunto de la información.

El segundo de los instrumentos que hemos utilizado es el análisis de documentos de las producciones científicas (artículos publicados) de las IP. Según Kerlinger (1986), el análisis de discurso es "un método de estudio y análisis de la comunicación de forma sistemática, objetiva y cuantitativa, con el fin de medir determinadas variables". El análisis del discurso implica una mirada analítica a un material procedente de hechos de la vida en entornos concretos (empírica) y a la vez maneja descripciones, explicaciones y narrativas para generar otras descripciones, explicaciones y narrativas (cualitativa). En esta investigación hemos estudiado a partir del análisis del discurso las producciones científicas de las directoras de los equipos de investigación SGR entrevistadas. Para ello, hemos partido del trabajo de Newman, Groom, Handelman, \& Pennebaker (2008) para el análisis del discurso de hombres y de mujeres en los Estados Unidos. Esta adaptación se ha hecho teniendo en cuenta las especificidades de la lengua castellana y los objetivos principales de nuestro proyecto de investigación. Se trataba de identificar si existen tendencias entre las mujeres profesoras universitarias de CCSS que permitan identificar una manera común a la hora de expresarse y cuáles son las características principales de su comportamiento discursivo. Más concretamente, nuestro instrumento de recogida de información investiga el discurso de las profesoras participantes desde tres dimensiones generales: la dimensión lingüística, la dimensión psicológica y la dimensión cognitiva.

Con respecto a la dimensión lingüística, nos interesa especialmente investigar el contexto y las características principales de la comunicación de las autoras con su público. En un segundo nivel intentamos identificar si las autoras utilizan un lenguaje científico neutro o sexista y si esto se hace intencionalmente o no. Finalmente, hacemos énfasis especial en las citas de las autoras (número de citas, citas con página, autocitas), puesto que consideramos este elemento significativo para analizar la confianza que sienten por su investigación.

En cuanto a la dimensión psicológica, hemos intentado investigar tres procesos psicológicos a la hora de

Tabla 2. Tópicos y descriptores de la investigación

\begin{tabular}{|c|c|}
\hline Tópicos & Descriptores \\
\hline $\begin{array}{l}\text { Antecedentes y contexto actual de } \\
\text { las profesionales }\end{array}$ & $\begin{array}{l}\text { - antigüedad en la investigación } \\
\text { - inspiración }\end{array}$ \\
\hline $\begin{array}{l}\text { El rol de la investigación y las otras } \\
\text { funciones académicas }\end{array}$ & $\begin{array}{l}\text { - relación con la gestión } \\
\text { - factores personales relativos a los aspectos personales } \\
\text { - incidencia de los sistemas de evaluación } \\
\text { - facilidad en el acceso a los recursos materiales }\end{array}$ \\
\hline Factores de éxito & $\begin{array}{l}\text { - factores organizativos internos } \\
\text { - factores organizativos externos }\end{array}$ \\
\hline Liderazgo del grupo de investigación & $\begin{array}{l}\text { - formación para el liderazgo } \\
\text { - fomento de la formación para la investigación } \\
\text { - mecanismos y criterios de selección de los miembros del equipo de investigación } \\
\text { - propuestas para mejorar la presencia de las mujeres en la investigación en CCSS }\end{array}$ \\
\hline $\begin{array}{l}\text { Temáticas en las CCSS } \\
\text { Metodologías propias } \\
\text { Difusión del conocimiento y la } \\
\text { investigación }\end{array}$ & $\begin{array}{l}\text { - la difusión de las investigaciones } \\
\text { - temáticas } \\
\text { - métodos }\end{array}$ \\
\hline
\end{tabular}


interpretar los artículos de nuestras participantes. En primer lugar, hemos querido analizar la aparición de emociones positivas de la autora, es decir identificar frases o palabras dónde la autora demuestra su satisfacción u optimismo para la investigación. Por otra parte, hemos buscado también la aparición de emociones negativas de las autoras, es decir de frases o palabras dónde se expresa su insatisfacción, tristeza, ansiedad sobre algunos aspectos de su investigación o de sus resultados. Finalmente, hemos considerado importante identificar la aparición de algunas sensaciones de la autora, es decir de información que ella transmite y que proviene de hechos o elementos que ha visto, sentido o escuchado.

Con respecto a la dimensión cognitiva, hemos intentado analizar cómo las investigadoras de nuestro estudio fundamentan su argumentación. Más concretamente, hemos querido estudiar la frecuencia de aparición de frases de causa y efecto, es decir el uso de conjunciones o modos conjuntivos (como por ejemplo: porque, de que, ya que, como, como que, por lo tanto, en efecto, etc.) para reforzar su argumentación. Después, hemos considerado importante investigar las frases de perspicacia que se utilizan, es decir frases dónde la autora refuerza su argumentación con frases o verbos como pienso, creo, me parece, según mi opinión, habría, etc. En el mismo contexto, hemos identificado las oraciones tentativas, dónde la autora fundamenta su argumentación con palabras y frases como casi, podría ser, quizás, no siempre, etc. Paralelamente, con las tentativas, hemos analizado la aparición de las frases ciertas donde la autora utiliza, para fomentar su argumentación, axiomas o palabras de certeza como por ejemplo: siempre, nunca, nada, en absoluto, etc. Finalmente, se ha hecho un análisis de los verbos cobertura (hedge verbos) de los artículos, es decir de palabras que se utilizan por la autora para dar "cobertura"/apoyo a su argumentación. Nos referimos a palabras que condicionan sus argumentos, como por ejemplo: "me parece", "según mi opinión", "podría ser", "según se espera", "posiblemente", etc. En la tabla 3 presentamos la tabla que utilizamos para recoger la información después de analizar los dieciocho artículos.

\section{RESULTADOS Y DISCUSIÓN}

En este apartado presentamos información extraída de las entrevistas en profundidad y del análisis del discurso para generar una discusión y exponer los resultados acerca de los intereses y preocupaciones de las IP de Grupos Consolidados en el área de las Ciencias Sociales de la UAB y de la UB. Ofrecemos un recorrido por los datos obtenidos porque los números en sí mismos son relevantes. Del total de los grupos de investigación (SGR), las investigadoras representan todavía una minoría en comparación con los hombres. Es necesario conocer las dificultades y facilidades de la carrera universitaria con las que se encuentran las

Tabla 3. Esquema general de la pauta de análisis de los artículos

\begin{tabular}{|l|l|}
\hline Título del artículo: \\
\hline Autora: & Ítems \\
\hline Dimensiones & No de palabras \\
\hline \multirow{4}{*}{ Lingüística } & Interrogantes \\
\cline { 2 - 2 } & Números (autores citados, datos, tablas numéricas,...) \\
\hline & Negaciones (no, nunca, nada, ni) \\
\hline & Preposiciones (y, con, incluso, o, sin, ni) \\
\hline Uso sexista o no del lenguaje (Genérico o no) \\
\hline \\
\hline Emociones positivas \\
\hline Emociones negativas \\
\hline Presentación de algunas sensaciones de la autora \\
\hline \\
\hline Uso de oraciones de causa y efecto \\
\hline Uso de oraciones de perspicacia \\
\hline Uso de oraciones tentativas \\
\hline Uso de oraciones ciertas \\
\hline Uso de verbos y frases cobertura \\
\hline
\end{tabular}


mujeres élite del área de CCSS y cuáles son sus intereses y preocupaciones en la investigación.

En primer lugar, y con la intención de focalizar el estudio, partimos de una primera aproximación a los datos ofrecidos por la Agencia de Gestión de Ayudas Universitarias y de Investigación sobre los grupos reconocidos por la Generalitat de Catalunya (SGR) (convocatoria 2009-2013) en el área de CCSS. Estos datos nos permiten afirmar que la mayoría de los SGR pertenecen a la Universidad Autónoma de Barcelona (UAB) y a la Universidad de Barcelona (UB). De igual manera, son estas dos universidades las que tienen mayor porcentaje de SGR liderados por mujeres, concretamente en la UAB 24 grupos y en la UB 36. En concreto, en la Universidad de Barcelona, teniendo en cuenta la distribución de los grupos de investigación reconocidos en el área de CCSS podemos afirmar objetivamente que el ámbito de Derecho posee el mayor número de SGR (12), seguido por el ámbito de Ciencias de la Educación (10) y el de Economía (9). En cuanto a la Universidad Autónoma de Barcelona, el mayor número de grupos consolidados pertenecen a los ámbitos de Economía y Educación (14) seguidos por Derecho, Psicología y Geografía. En la tabla 4 se muestran los datos correspondientes al número de SGR liderados por mujeres en ambas universidades catalanas.

Como ya hemos dicho, a pesar del soporte legal obtenido para acceder de forma generalizada a la Universidad, para las mujeres continúan existiendo serias dificultades para acceder a lugares relevantes y de poder en el mundo académico, tal y como se muestra en el cuadro anterior. El número de mujeres que coordinan un SGR es bastante más bajo con respecto a los grupos liderados por hombres (por ejemplo, en la Universidad de Barcelona sólo el $27,7 \%$ de los SGR están liderados por mujeres, según datos del GREC, 2007) excepto en los ámbitos de Geografía y Derecho de la UAB.
Pero si lo que nos interesa es conocer cuáles son los temas y los problemas de investigación de las mujeres que lideran SGR en la UB y UAB y no tanto el número de mujeres IP debemos centrarnos en conocer las temáticas generales de investigación del SGR. En la tabla 5 se muestran los SGR liderados por mujeres en la UB y en la UAB.

Como podemos apreciar de la tabla 5, la mayoría de los SGR dejan entrever que la investigación se centra en tópicos propios del área de conocimiento y del ámbito específico. A pesar de ello, según la información obtenida en las entrevistas, algunas de las informantes afirmaban que a las mujeres les interesa más los temas humanísticos y aquellos temas que tienen que ver con hechos más reales:

"Por lo que he visto en mi vida los hombres son mucho más abstractos en mi área a la hora de investigar que las mujeres. Mis temas son mucho más humanos tocan más a la realidad, mientras que mis colegas tocan temas muy abstractos para hacer teorías generales. Esta es la percepción que tengo de manera general, pero no en mi grupo en donde hay más bien una mezcla, porque hay mujeres que tienen teoría más general y tengo un titular de Madrid que es mucho más concreto; por lo que actualmente en mi grupo no veo yo esa diferencia" (A1).

Así también observamos que existen SGR liderados por mujeres donde el nombre del grupo muestra explícitamente que las temáticas de los estudios giran en torno a tópicos relacionados con el género. En relación a esto, es decir, al interés por estudios de género, y teniendo en consideración todos los SGR liderados por mujeres en todos los ámbitos de las CCSS en ambas universidades, nos encontramos con que en la UB existen 2 grupos en los que su nombre alude específicamente a estudios de género. En la UAB la situación es similar, existen tres grupos de investigación que el nombre del grupo hace referencia explícita a estudios de género (tabla 6).

Tabla 4. Distribución de los grupos por universidades y género

\begin{tabular}{|c|c|c|c|}
\hline UNIVERSIDAD & ÁMBITOS & LIDERADOS POR MUJERES & TOTAL DE GRUPOS SGR \\
\hline \multirow{3}{*}{ Universidad de Barcelona } & Derecho & 3 & 12 \\
\cline { 2 - 4 } & Educación & 4 & 10 \\
\cline { 2 - 4 } & Economía & 1 & 9 \\
\hline \multirow{3}{*}{$\begin{array}{c}\text { Universidad Autónoma de } \\
\text { Barcelona }\end{array}$} & Economía & 7 & 14 \\
\cline { 2 - 4 } & Educación & 5 & 9 \\
\cline { 2 - 4 } & Derecho & 2 & 7 \\
\cline { 2 - 4 } & Psicología & 4 & 82 \\
\hline
\end{tabular}


Tabla 5. SGR liderados por mujeres en Ciencias Sociales en la UAB y en la UB

\begin{tabular}{|c|c|}
\hline Derecho & $\begin{array}{ll}\text { 1. DERECHO CIVIL CONSTITUCIONAL Y DERECHO CATALÁN (UB) } \\
\text { 2. GRUPO INTERUNIVERSITARIO DE DERECHOS CULTURALES Y DIVERSIDAD (GIDD) (UB) } \\
\text { 3. ÁREA DE DERECHO INTERNACIONAL PRIVADO DE LA UNIVERSDAD DE BARCELONA (UB) } \\
\text { 4. DERECHO Y SOCIEDAD DESDE LA PERSPECTIVA DE GÉNERO (UAB) } \\
\text { 5. DERECHO CONSTITUCIONAL EUROPEO (UAB) } \\
\text { 6. ESTUDIOS INTERNACIONALES Y EUROPEOS DEL INSTITUTO DE DERECHO Y TECNOLOGÍA (UAB) } \\
\text { 7. ELITES Y PARTIDOS POLÍTICOS (UAB) } \\
\text { 8. ESTUDIOS FEDERALES Y AUTONÓMICOS (UAB) }\end{array}$ \\
\hline $\begin{array}{l}\text { Economía } \\
\text { Aplicada }\end{array}$ & $\begin{array}{l}\text { 1. CREATIVIDAD, INNOVACIÓN Y TRANSFORMACIÓN URBANA (UB) } \\
\text { 2. RIESGO EN FINANZAS Y SEGUROS (UB) } \\
\text { 3. NIVELES DE VIDA Y MEDIO AMBIENTE (UB) }\end{array}$ \\
\hline Educación & $\begin{array}{l}\text { 1. SUBJECTIVIDADES Y ENTORNOS EDUCATIVOS CONTEMPORÁNEOS (ESBRINA) (UB) } \\
\text { 2. GRUPO DE INVESTIGACIÓN EN EDUCACIÓN INTERCULTURAL (UB) } \\
\text { 3. ENTORNOS Y MATERIALES PARA EL APRENDIZAJE (UB) } \\
\text { 4. GRUPO EDUCACIÓN SUPERADORA DE DESIGUALDADES (UB) } \\
\text { 5. LENGUAJE Y ENSEÑANZA DE LAS CIENCIAS- (UAB) } \\
\text { 6. EDUCACIÓN MATEMÁTICA Y CONTEXTO SOCIOCULTURAL - EMICS (UAB) } \\
\text { 7. EDUCACIÓN Y SEGURIDAD VIAL (UAB) } \\
\text { 8. ENSEÑANZA E INTERACCIÓN PLURILINGÜES (UAB) } \\
\text { 9. INNOVACIÓN DIDÁCTICA Y VALORES EN LA EDUCACIÓN FÍSICA Y EL DEPORTE (UAB) } \\
\text { 10. INVESTIGACIÓN MUSICAL Y EDUCACIÓN (UAB) } \\
\text { 11. LITERATURA INFANTIL Y JUVENIL Y EDUCACIÓN LITERARIA (UAB) }\end{array}$ \\
\hline $\begin{array}{l}\text { Sociología y } \\
\text { Política }\end{array}$ & $\begin{array}{l}\text { 1. GRUPO DE INVESTIGACIÓN EN GÉNERO, IDENTIDAD Y SOCIEDAD (UB) } \\
\text { 2. GRUPO DE INVESTIGACIÓN EN TERRITORIO, POBLACIÓN Y CIUDADANÍA (UB) } \\
\text { 3. COPOLIS. BIENESTAR COMUNIDAD Y CONTROL SOCIAL (UB) } \\
\text { 4. GRUPO DE INVESTIGACIÓN E INNOVACIÓN EN TRABAJO SOCIAL (GRITS) (UB) } \\
\text { 5. ESTUDIOS DE INMIGRACIÓN Y MINORÍAS ÉTNICAS (UAB) }\end{array}$ \\
\hline Geografía & $\begin{array}{l}\text { 1. } \text { ESTUDIOS DE LA POBLACIÓN (UAB) } \\
\text { 2. INVESTIGACIÓN DE GEOGRAFÍA Y GÉNERO (UAB) } \\
\text { 3. TURISMO Y DINÁMICAS SOCIO-TERRITORIALES (UAB) } \\
\text { 4. RECURSOS COSTEROS, TERRITORIOS Y PAISAJIISTICOS (UAB) }\end{array}$ \\
\hline Psicología & $\begin{array}{l}\text { 1. ESTUDIOS SOCIALES Y DE GÉNERO DE PODER Y LA SUBJECTIVIDAD (UAB) } \\
\text { 2. INVESTIGACIÓN PSICOEDUCATIVA SOBRE LA SORDERA Y OTRAS DIFICULTADES } \\
\text { COMUNICATIVAS (UAB) }\end{array}$ \\
\hline
\end{tabular}

Tabla 6. Relación de grupos de investigación que tienen el género como tópico de investigación

\begin{tabular}{|l|l|}
\hline \multicolumn{2}{|l|}{ UNIVERSIDAD DE BARCELONA } \\
\hline Educación & Grupo educación superadora de las desigualdades \\
\hline Sociología y Política & Grupo de investigación en género, identidad y sociedad \\
\hline UNIVERSIDAD AUTÓNOMA DE BARCELONA \\
\hline Geografía & Investigación de Geografía y Género \\
\hline Psicología & Estudios Sociales y de Género de Poder y la Subjetividad \\
\hline Derecho & Antígona, Derecho y Sociedad en Perspectiva de Género \\
\hline
\end{tabular}


$Y$ es que, efectivamente, tal y como afirma otra de las participantes, son las mujeres las que se interesan más por los tópicos que tienen que ver precisamente con el estudio del género o sobre temáticas relacionadas con la mujer.

"Sí que es cierto que hay algunos tópicos que tocan más de cerca las mujeres y entonces es normal que al principio exista una preocupación no científica, digamos curiosidad o motivación por un tema, aunque luego se acabe convirtiendo en un interés científico. Por ejemplo, es normal que cuestiones de filosofía feminista hayan sido estudiadas primero por mujeres". (A3)

En concreto, de los cinco SGR liderados por mujeres y que según el nombre del grupo induce a pensar que investigan temas relacionados con el género, es posible a través de la difusión de la investigación (subproyectos, artículos), afirmar que efectivamente trabajan temáticas relacionadas con la mujer. Concretamente, entre sus objetivos está la creación de espacios, recursos e iniciativas sobre mujeres (cada una desde sus disciplinas, ya sea desde el Derecho o desde la Geografía); o el aumento de la capacidad de integración de las mujeres.

A partir del documento "Relación de los grupos de investigación reconocidos por la Generalitat de Catalunya (SGR-DGR 2009-2013)", en donde se menciona y se distribuye toda la relación de SGR de las universidades catalanas por 3 grandes bloques, es posible observar que 4 de estos últimos 5 SGR se ubican en el bloque de: "Retos para las personas y la sociedad" y en concreto dentro del subbloque de "Cohesión social y gestión de la complicidad social para generar oportunidades", dato que confirma nuestra afirmación. Aún así y a pesar de que la mayoría de las académicas entrevistadas hiciesen mención a la sensibilidad o motivación por el estudio de cuestiones relativas al género, o incluso a la preocupación por temas más humanos y cercanos a la realidad social, hemos de ser prudentes al afirmar que la variable género sea una variable determinante en la selección de determinadas temáticas o que induzcan a metodologías de investigación diferentes.

No hay unanimidad en las informantes en pensar que el hecho diferenciador del género sea determinante para la elección de una temática u otra en investigación y que ésta a su vez sea significativamente diferente a las temáticas elegidas por los varones.

"Yo creo que en la formación de las personas, sus intereses $y$, sobre todo, su trayectoria vital condiciona su investigación. Por tanto no creo que sea por el hecho físico del género, si fuese así significaría que no podría haber grupos de hombres y mujeres investigando un tema común". (A4)

Lo que sí es posible afirmar a partir de las entrevistas realizadas es que las mujeres apelan a la necesidad de cambio social en el contenido de los roles asociados al género. Mientras no haya equidad funcional en lo relativo al mundo familiar, más allá de lo profesional, seguirá siendo difícil para las mujeres tener las mismas oportunidades laborales que los hombres.

"Creo que es un poco complicado, no solamente es tarea de la mujer, sino que también hay tarea para los hombres investigadores, que son los que por tradición muchas veces están liderando los equipos de investigación". (E5)

"Yo sigo pensando que el modelo familiar en el que hay adultos que están muy presentes y que comparten muchas cosas, es válido. Lo que debería cambiar es el valor que se da al rol de uno y de otro". (E6)

En cualquier caso, uno de los pasos que a nivel social debe darse con mayor contundencia es el aseguramiento de sistemas de conciliación de la vida familiar y laboral con el objetivo de evitar que las académicas deban elegir entre su familia y su carrera ya que ambos escenarios vitales son irreconciliables.

"Sólo veo que debe haber conciliación entre familia y trabajo, pero me parece que eso no es tanto un tema de la Universidad o de las organizaciones públicas, sino del sistema. A veces lo que es difícil es eso: conciliar la familia y el trabajo. Me parece que en el caso de las ciencias sociales, no sé si hay reglas de paridad o cuotas, al momento de conceder estos grupos, pero me parece que más que ejercer la paridad hay que colaborar en leyes para la conciliación". (A1)

Existen circunstancias individuales, intereses, preocupaciones y elementos que o bien favorecen o dificultan la producción científica de las mujeres en la Universidad. Las informantes coinciden en afirmar que el hecho de ser mujer es más una dificultad que una ventaja para trabajar en la Universidad y sobre todo, a la hora de liderar grupos de investigación consolidados que en la mayoría de los casos están compuestos por más hombres que mujeres. Las barreras parecen ser muchas:

"Es un campo en que a pesar de que hay muchas estudiantes, muchas doctorandas y muchas mujeres iniciando la carrera, tenemos sólo 4 catedráticas en toda España que ahora están desesperadas con las oposiciones de cátedra porque tienen que estar recorriéndose toda España para ir a los tribunales y, por tanto, estoy en un campo que no puedo decir que sea especialmente 'friendly' para las mujeres". (E1)

"A veces por comodidad no harías cosas o no competirías o no te presentarías a cosas pero crees que las tienes que hacer ¿por qué? Bueno, porque no hay mujeres o no hay candidatas o te piden hacer algo que es interesante en general, para las mujeres en general, o para intentar mejorar la igualdad de género en general, y esto es importante; por comodidad muchísimas veces te quedarías en tu casa, estás muy cansada y muy aburrida". (E1) 
Es por ello, que entre los intereses manifestados por algunas de las académicas está la creación de redes y espacios de convivencia desde donde poder crear sinergias con la finalidad de darse apoyo mutuo.

"Las mujeres suelen establecer más redes de ayuda, porque lo han hecho siempre, y por tanto también lo trasladan y la red de ayuda siempre beneficia al grupo, y si beneficia al grupo beneficia a los individuos, pero esa es una visión que no está muy institucionalizada en la Universidad". (E3)

"Yo trabajo sobre cuestiones de género, es decir, trabajo sobre el Mercado de Trabajo y Diferencias de Género en el Mercado de Trabajo en perspectiva histórica; entonces eso hace que se creen apoyos especiales que me han venido principalmente por mujeres que han trabajado en ese campo y son exclusivamente mujeres". (E1)

La mayor presencia de mujeres en el mundo académico ha ido acompañada de una mayor dedicación de éstas a determinadas disciplinas consideradas más "femeninas" (Humanidades y CCSS y jurídicas). Es especialmente destacable que el género sigue aún condicionando la elección por determinadas áreas de conocimiento.
"En nuestro grupo la mayoría de las personas somos mujeres, claro que en el campo de las ciencias sociales eso es bastante común. Aunque sí creo que la agenda sentimental de las mujeres es siempre diferente a la de los hombres. Porqué, claro, un hombre que se quiere dedicar a la investigación, se autoimpone menos responsabilidades familiares, y pues claro que las mujeres nos solemos auto imponer más. Yo, en este momento, como decía una persona a la que le hicimos una historia de vida y que es catedrática de matemáticas, tomando en cuenta que sólo el $9 \%$ de los catedráticos en matemáticas son mujeres, ella decía que: "el que quiere hacer camino, lo hace" no importando si es hombre o mujer" (A2)

Sin embargo, es igualmente relevante ver como aún existe una brecha importante en relación a la permanencia en las categorías inferiores del escalafón académico (Informe Nacional, España, 2011).

Para el análisis del discurso de los 18 artículos, utilizamos la pauta adaptada de Newman et al. (2008). Este análisis nos ha permitido identificar la manera de expresarse y las principales características lingüísticas, psicológicas y cognitivas de la expresión escrita de las producciones de las profesoras (tabla 7).

Tabla 7. Textos analizados de las autoras

\section{Títulos de los artículos analizados}

1. Nota a propósito de la utilización de Needham por Ortuño.

2. Etnografía, brujería y tensión social: las tribulaciones de Marwick para poner a prueba la tesis obstétrica matrilineal.

3. Relativismos, realismo y el ámbito de aplicación del conocimiento antropológico.

4. Los observatorios de la sociedad de la información: evaluación o política de promoción de las TIC en educación.

5. Los desafíos de la educación desde la óptica de la OCDE.

6. De TIC a TAC, el difícil tránsito de una vocal.

7. Posibilidades y relevancia de la observación sistemática por el profesional de la psicología.

8. Evaluación de programas desde la metodología cualitativa.

9. Como apresar las competencias del bebé mediante una aplicación de la metodología observacional.

10. Una nueva etapa en la protección internacional de adultos.

11. La regulación de la adopción en España: examen particular de la adopción internacional.

12. Un espacio europeo de libertad, seguridad y justicia: la cooperación en el ámbito civil.

13. Emigración interior, emigración exterior.

14. El concepto de igualdad ante el mercado.

15. Acerca de la modernización, la modernidad y el riesgo.

16. Barcelona de principis del segle XX a partir de les fonts literàries de dones.

17. Estructura familiar i treball de la dona a l'agricultura: el cas d'Osona i el Baix Empordà.

18. Turismo religioso en Montserrat: Montaña de fe, montaña de turismo. 
Con respecto a la dimensión lingüística, hemos identificado una ausencia general de preguntas y auto-citaciones en los trabajos de investigación de las mujeres profesoras que participaron en nuestra investigación. Por otro lado, se utilizan frases ampliamente inclusivas y conjunciones. Con respecto a la dimensión psicológica, podemos decir que las mujeres que coordinan los grupos de investigación en CCSS y que participaron en nuestro estudio parecen preferir la expresión de emociones positivas y optimistas que las negativas en sus trabajos de investigación. Además, no identificamos ninguna tendencia específica relativa al uso de un lenguaje sexista. Finalmente, con respecto a la dimensión cognitiva, no podemos encontrar ninguna tendencia o patrón relacionado con la construcción de frases o párrafos. Cada participante parece seguir su escritura personal y sus preferencias sintácticas, que no están relacionadas con su disciplina o ámbito de conocimiento.

En este contexto, entendemos que se necesita un análisis más preciso sobre las dimensiones anteriores el cual, según Newman et al. (2008), debe contener no sólo datos numéricos sobre las palabras utilizadas en los artículos y una descripción superficial del estilo de la expresión escrita de las participantes, sino más bien un análisis del estilo literario y de las dimensiones cognitiva y psicológica. Este estudio no puede ofrecer resultados finales todavía, pero abre una nueva ventana para hacer frente a la cuestión de género en la escritura académica y se puede utilizar como punto de partida para un nuevo diálogo entre expertos e instituciones con experiencia en esta área científica.

\section{CONCLUSIONES}

De los resultados obtenidos podemos anunciar tendencias más que conclusiones. Sólo una cuarta parte de los grupos son liderados por mujeres y éstas parece que tienen unas condiciones especiales y que están ahí porque siguen las pautas investigadoras al uso: metodologías de investigación propias de la disciplina, estilos de redacción de los artículos de investigación influidos por la tradición,... Parece observarse como rasgo diferenciador del género femenino una mayor preocupación por la formación del equipo de investigadores y por el clima del grupo.

Se confirma la hipótesis de que la realidad del profesorado universitario femenino es diferente y que buena parte de esta diferencia está marcada por la construcción social del género. Asímismo, se confirma que el interés por la investigación relacionada con el género corresponde aún a la mujer y que las dificultades para acceder a la élite académica sigue patente. Los datos existentes sobre la situación de la mujer en la Ciencia y la Universidad ponen en evidencia las dimensiones de discriminación y desigualdad en el momento de la promoción profesional concluyendo que la plena incorporación de la mujer a la Universidad no ha implicado su promoción en el mundo académico.
Para comprender y diagnosticar la situación de las mujeres investigadoras en CCSS en las dos grandes universidades catalanas, hemos recurrido en primer lugar a una comparación de tipo estadístico. En este sentido los números son relevantes, puesto que del total de los grupos de investigación, las investigadoras representan todavía una minoría en comparación con los hombres. El análisis de los grupos de investigación consolidados nos ayuda a acercarnos al campo del estudio de las élites académicas. Se trata de académicos y académicas que han llegado a un nivel de consolidación de la carrera profesional investigadora. Como apuntan otros estudios (ver por ejemplo García de León, 2001) el estudio de las élites forma parte de la sociología del poder, tema sustancial en CCSS y llama la atención sobre cómo se distribuye el poder en la Ciencia y aunque la universidad actual catalana ha conocido en los últimos años, cambios importantes, este proceso no está acompañado por un cambio en la distribución igualitaria entre hombres y mujeres en el ámbito de la investigación en general y en ciencias sociales en especial.

Se ha presentado una primera aproximación al estudio del discurso de las mujeres en CCSS, pero todavía estamos lejos de poder ofrecer conclusiones definitivas. Con respecto al instrumento utilizado de Newman y otras (2008) podemos decir que es útil como punto de partida aunque ha requerido de sucesivos ajustes debido a las diferencias en el lenguaje y el contexto de la aplicación (la Universidad).

Además, consideramos que se debe utilizar con una muestra más grande de textos académicos para poder extraer resultados significativos. En esto ayudaría mucho el uso de un programa de ordenador especializado en el análisis del discurso.

Podemos decir que el instrumento escogido para el análisis del discurso fue una buena herramienta para analizar las tendencias/preferencias lingüísticas entre las mujeres profesoras universitarias de CCSS, porque nos ha permitido identificar su manera de expresarse y las principales características lingüísticas, psicológicas y cognitivas de su expresión escrita.

En relación a los intereses y las preocupaciones las participantes manifiestan que lo que más les motiva es la investigación en sí misma. Las problemáticas a las que se enfrentan las investigadoras en el ámbito de las Ciencias Sociales son muy parecidas a las de sus colegas masculinos (sistemas de evaluación externa, carencia de financiación de la investigación lineal, plantillas poco estables, etc.). Se preocupan por seguir manteniendo las relaciones internacionales que han ido fomentando a lo largo de sus carreras académicas. Asistir a congresos y establecer contactos interuniversitarios, en relación a los temas de investigación, son tareas que priorizan. 
Otro tema del cual se ocupan las participantes es en seguir formándose en aquello que investigan y en cómo investigan. Entienden que esta formación va unida a menudo a la asistencia a congresos y al establecimiento de vínculos académicos. A las investigadoras les preocupa que haya pocas mujeres que lideran equipos de investigación de renombre y que faltan modelos femeninos en los cuales las jóvenes puedan inspirarse. Consideran que para tener una buena producción científica el/la estudiante de doctorado debe poder contar con una persona que en los inicios de la carrera académica le dé orientación en la toma de decisiones y apoyo.

\section{NOTAS}

1 Tomàs. M. (Coordinadora); Bernabeu D.; Castro, D.; Duran, M.M.; Ion, G.; Vlachopoulos, D.; Mentado, T.; Colaboradora: Rebollar, E.M. (2001) Informe: La investigació acadèmica en Ciències Socials des d'una perspectiva de gènere. Estudi de cas a la UAB i a la UB. I'Institut de la Dona, Generalitat de Catalunya. Referencia U-45/10.

\section{BIBLIOGRAFÍA}

Agència de gestió d'ajuts universitaris i de recerca de la generalitat de Catalunya, Departament d'innovació universitats i empreses, Relació per focus dels grups de recerca reconeguts per la Generalitat de Catalunya (SGR-DGR 2009-2013). http://www10.gencat.cat/agaur_web/ generados/catala/home/recurs/doc/ focus_sgr_grups.pdf

Botella, J. (2010). Som diferents els de LLetres? Ponencia del grup 4. Taller L'avaluació de la recerca en Humanitats i Ciencies Socials UB, 28 i 29 gener. AQU.

Consejo Superior de Investigaciones Científicas (CSIC) (2009), informe Mujeres y Ciencia "Mujeres Investigadoras 2009". (http://www.csic.es/web/guest/mujeres-ilustres

Del Pozo, J.M. (2010). La evaluación de conocimiento Humanístico y Social. Ponencia inaugural. Taller L'avaluació de la recerca en Humanitats i Ciencies Socials UB, 28 i 29 gener. AQU.

Guil, A. (2004). Estudios específicos sobre mujeres y ciencia en Andalucía. Informe para el grupo de expertas Mujer y Ciencia FECYT, Spanish Foundation for Science and Technology, Madrid.

García de Cortázar, M. L.; García de León, M. A. (1997). Mujeres en minoría: una investigación sociológica sobre las catedráticas de universidad en España. Madrid: Centro de Investigaciones Sociológicas.

García de León, M. A. (2001) (Coord.). Las académicas. Madrid: Ministerio de Trabajo y Asuntos Sociales.
Grup educació superadora de desigualtats. http://www.ub.edu/web/ub/es/ recerca_innovacio/recerca_a_la_UB/grups/ fitxa/G/CREPERAD/index.html

Grup de recerca en gènere, identitat i societat. http://www.ub.edu/web/ub/es/ recerca_innovacio/recerca_a_la_UB/ grups/fitxa/G/GENICULE/equip/nvestigador/index.html

Grup de Recerca de Geografia i Gènere. http://geografia.uab.es/ genere/index.php? option=com_ content\&view=frontpage\&/temid=1

Grup de recerca Estudis Socials i de Gènere de Poder i la Subjectivitat. http://psicologiasocial. uab.es/ca/node/181.

Grup de recerca Antígona, Dret i Societat en Perspectiva de Gènere. http://antigona.uab.cat/.

Kerlinger, F. N. (1986). Foundations of behavioral research (3ed 2d.). New York: Holt, Rinechart and Winston.

Ley Orgánica 3/2007, de 22 de marzo, para la igualdad efectiva entre mujeres $y$ hombres.

Ley Orgánica 4/2007 de 12 de abril, que modifica la Ley de Universidades.

Martín Lou, M. A. (2002). Área de Humanidades y Ciencias Sociales: una visión en el tiempo. Arbor CLXXII, 679-680 (JulioAgosto 2002), pp. 537-548.

Miqueo, C.; Barral, M. J. y Magallón, C. (eds.) (2008). Estudios Iberoamericanos de género en ciencia, tecnología y salud (GENCIBER). Zaragoza: Prensas Universitarias de Zaragoza, 135-142.
Montané, A y Beltrán, J. (coords.) (2011). Informe Nacional. Políticas de cohesión y equidad en la educación superior en España. Programa Marco Interuniversitario para una política de equidad y cohesión social en la educación superior. EuropeAid/129877/C/ACT/Multi.

Newman, M. L.; Groom, C.; Handelman, L. y Pennebaker, J. (2008): Gender Differences in language Use: An Analysis of 14000 Text Samples. Discourse Processes, 45, 211-236.

Panchón, C. (2010). Principals problemes en l'avaluació de la recerca en Humanitats i en Ciències Socials. Ponència del grup 3. Taller L'avaluació de la recerca en Humanitats i Ciències Socials UB, 28 i 29 gener. AQU.

Pérez Sedeño, E. (dir.) (2003). La situación de las mujeres en el sistema educativo de ciencia y tecnología en España y su contexto internacional. Programa de análisis y estudios de acciones destinadas a la mejora de la calidad de la enseñanza superior y de actividades del profesorado universitario (REF: S2/ EA2003---0031). Disponible en: http:// www.centrorecursos.com/mec/ayudas/CasaVer.asp?P=29 33

Subirats, J. (2010). Reflexió sobre l'avaluació de la recerca. Ponència del grup 4 . Taller L'avaluació de la recerca en Humanitats i Ciencies Socials UB, 28 i 29 gener. AQU.

Unidad de Mujeres y Ciencia (UMYC) http:// www.micinn.es/portal/site/MICINN / menuitem.7eeac5cd345b4f34f09dfd10 01432ea0/?vgnextoid =e218c5aa16493 210VgnVCM1000001d04140aRCRD 